\title{
Distributions of Parameters and Features of Multiple Bond Ruptures in Force Spectroscopy by Atomic Force Microscopy
}

\author{
Senli Guo, Nan Li, Nimit Lad, Shivam Desai, and Boris B. Akhremitchev* \\ Department of Chemistry, Duke University, Durham, North Carolina 27708
}

Received: January 20, 2010; Revised Manuscript Received: April 19, 2010

\begin{abstract}
Force spectroscopy measurement of rupture forces of bound molecules becomes an important physicochemical tool in characterizing intermolecular interactions. Atomic force microscopy (AFM) measurements are among the most common approaches in implementation of this technique. Kinetic information about the molecular bond under study is usually extracted assuming that the detected rupture force comes from rupturing of a single bond. However, multiple bond ruptures might occur in experiments. In this article, we consider how the presence of multiple bonds is manifested in the distribution of parameters that are typically extracted in force spectroscopy experiments. Of particular interest here are the distributions of rupture forces and Kuhn lengths of polymeric tethers. We show that multiple bond ruptures might contribute to the measured distributions even when these distributions have a well-defined single peak. Also, we consider how the probability to form multiple bonds depends on probe velocity. The developed analytical models are applied to experimental data of biotin-streptavidin ruptures. The velocity dependence of the amplitude of high force tail supports the hypothesis of multiple bond nature of the measured high forces.
\end{abstract}

\section{Introduction}

The dissociation rate of a molecular bond in the presence of an external force that pulls molecules apart depends on the shape of the potential of mean force. ${ }^{1-6}$ This relationship makes force spectroscopy a valuable tool in the quantitative characterization of the energy landscape that governs interactions between molecules. In force spectroscopy, kinetic information about the dissociation reaction is obtained by measuring the dependence of rupture forces on the loading rate. The data analysis is usually based on approximations derived from the one-dimensional Kramer's reaction rate theory. Existing approaches can be categorized into approaches that consider dissociation from a well of the free energy potential with a specific shape (modelbased approaches $)^{7-10}$ and approaches that do not use a priori knowledge about shape of the free energy potential (modelfree approaches). ${ }^{11-13}$ Majority of the data analysis approaches are developed assuming that the experimentally registered rupture forces correspond to ruptures of single bonds. This assumption is essential in application of the model-free approaches. On the other hand, the model-based approaches can be adopted to explicitly include ruptures from multiple bonds. ${ }^{14-16}$ Therefore, proper application of the force spectroscopy technique requires that a distinction should be made between single and multiple bond ruptures in the data analysis. Atomic force microscopy (AFM) is one of the most common techniques in conducting force spectroscopy measurements. Experimental features in AFM measurements that distinguish single-bond ruptures from multiple-bond ruptures are the main subject of this article.

There are several experimental approaches where the singlemolecule nature of measured interactions is established using a mechanical "fingerprint" feature in the measured force-separation dependencies. This fingerprint is often obtained from mechanical unfolding of "polyproteins", ${ }^{17-20}$ or it naturally occurs during

* To whom correspondence should be addressed. E-mail: boris.a@ duke.edu. Phone: (919) 660-1648. Fax: (919) 660-1605. rupture of molecular bonds. ${ }^{21,22}$ Stretching of a polyprotein with individual "monomers" corresponding to folded globular proteins shows the restoring force that exhibits a sawtooth-like rupture pattern. In this pattern, the peak force is interpreted as unfolding of individual domains, and the spacing between the peaks corresponds to the gain in length of unfolded regions. ${ }^{17-20}$ In such experiments, stretching of two chains in parallel might be manifested by a sawtooth pattern with much higher unfolding forces and with mis-spacing of adjacent unfolding peaks. ${ }^{18}$ However, a characteristic sawtooth-like pattern can be also observed from pulling on two chains in parallel. ${ }^{23}$ In such case, analysis of the magnitude of rupture forces and persistence lengths of unfolded regions can be used to distinguish between single and multiple chains. Also, a characteristic rupture pattern is observed in rupture of the molecular bond responsible for initial polymerization of fibrin. ${ }^{21}$ For these interactions, it is suggested that the characteristic pattern consisting of two, four sequential ruptures originates from unfolding of a globular D domain of fibrin. A single-molecule nature of these ruptures is supported by the analysis of distributions of distances between rupture events and by the detailed kinetic analysis of distributions of rupture forces. ${ }^{22}$ Obvious fingerprint patterns are not always available in force spectroscopy experiments that study dissociation of molecular bonds. Therefore, a distinction between ruptures of single bonds and ruptures of multiple bonds is often ambiguous in these experiments. It might be suggested that if molecules are distributed on surfaces randomly and the overall detection probability is low then the probability of multiple ruptures is negligible. However, this argument cannot be used when either of the interacting molecules is multivalent or if the molecules have a propensity to cluster during the tip-sample preparation as explained below.

Existing approaches to analyze multiple ruptures use different assumptions about the magnitude of the resulting rupture force. One model is based on the assumption that measured rupture forces are "quantized" as multiples of rupture forces of single bonds. ${ }^{24,25} \mathrm{~A}$ kinetic model of a large number of parallel bonds 
indicates that rupture forces in the force-ramp experiments are expected to be proportional to the number of bonds. ${ }^{26}$ However, detailed kinetic models indicate that for two bonds that are subjected to equal pulling forces the total most probable rupture force is lower than the sum of two individual most probable forces measured in single-molecule experiments. ${ }^{14,27,28}$ Moreover, if forces on two bonds are not equal, the resulting most probable rupture force becomes even lower. ${ }^{16,29}$ In this article, we use an analytical model ${ }^{16}$ that does not make an assumption that the forces applied to separate bonds are equal.

Analysis of kinetic parameters in force spectroscopy experiments is based on measured distributions of rupture forces. These distributions and distributions of Kuhn lengths (or persistence lengths) of polymeric tethers are usually obtained in the initial analysis of force spectroscopy data. Below we describe how these distributions are affected by the presence of multiple ruptures. In addition, effects of multiple ruptures can be smeared by other experimental factors. Therefore, our analysis is based on analytical models that consider effects of several factors such as off-apex tether attachment, off-normal pulling geometry, and force baseline offset. We also consider how the probability to form multiple bonds depends on probe velocity. Using these models, we analyze the results from force spectroscopy measurements of interaction between biotin ligand and streptavidin receptor. It these measurements, the ligand is covalently tethered to the AFM probe by a polymeric linker and the receptor is covalently attached to the substrate. This analysis is followed by discussion of noted agreements and disagreements of experimental results with theoretical predictions.

\section{Theoretical Models}

2.1. Distribution of Rupture Forces in Single-Molecule Measurements with Constant Loading Rate. The Bell-Evans phenomenological model ${ }^{1,7}$ that is often used in analysis of rupture forces predicts that distribution of rupture forces is skewed toward lower forces and that there should be no forces considerably higher than the most probable force. ${ }^{29}$ However, such high forces are often measured experimentally. Earlier we have suggested that the "tail" of high rupture forces might be amanifestation of multipleruptures that occurnearly simultaneously. ${ }^{16,29}$ On the other hand, it has been suggested that these high rupture forces might come from rupture of one bond if the energy landscape has inner barriers of a particular height. ${ }^{30}$ We note that high rupture forces might be also obtained from free energy potentials with only one well without internal barriers if an assumption of the Bell-Evans model about the stationary position of a transition state is eliminated (as explained below). Therefore, we first consider the expected distribution of experimentally measured parameters when a single bond is being ruptured in AFM experiments.

In our theoretical description below, we consider that dissociation of molecules subjected to pulling force follows the first-order kinetics. A more detailed theoretical description of force spectroscopy can be found in other publications. ${ }^{1-9,31-34}$ The distribution of rupture forces in experiments with monotonically increasing pulling force is described by the probability density function (PDF). This function $p(F)$ is related to the bond survival probability by ${ }^{31}$

$$
p(F)=\frac{k(F)}{v_{\mathrm{F}}(F)} S(F)
$$

Here $S(F)$ is the bond survival probability at force $F$, assuming that pulling starts with the intact bond at force zero, $k(F)$ is the dissociation rate as a function of pulling force, and $v_{\mathrm{F}}=\mathrm{d} F / \mathrm{d} t$ is the loading rate that is a constant or an increasing function of time. For pulling force monotonically increasing from zero to force $F$, the survival probability can be calculated by ${ }^{31}$

$$
S(F)=\exp \left[-\int_{0}^{F} \mathrm{~d} f k(f) v_{\mathrm{F}}^{-1}(f)\right]
$$

In this article, we consider cases where a dissociation reaction can be described reasonably accurately by pulling along a onedimensional potential of mean force (PMF). There are several analytical models that provide closed-form expressions for $k(F)$ by making assumptions about the shape of PMF. ${ }^{1,8-10}$ The Bell-Evans model ${ }^{1,7}$ assumes that distance between positions of the equilibrium state and the transition state $\left(x^{\ddagger}\right)$ is independent of pulling force. Therefore, the dissociation rate as a function of pulling force is ${ }^{35}$

$$
k(F)=k_{0} \exp \left(F x^{\ddagger} / k_{\mathrm{B}} T\right)
$$

Here $k_{0}$ is the dissociation rate at zero force, $k_{\mathrm{B}} T$ is the thermal energy, and $x^{\ddagger}$ is the distance between the equilibrium state and the transition state (henceforth called "barrier width" for brevity). For kinetic experiments with constant loading rate $v_{\mathrm{F}}$, the PDF predicted by this model is

$$
p_{\mathrm{BE}}(F)=\frac{k_{0}}{v_{\mathrm{F}}} \exp \left(\frac{F}{F^{\ddagger}}-\frac{k_{0} F^{\ddagger}}{v_{\mathrm{F}}}\left(\mathrm{e}^{F / F^{\ddagger}}-1\right)\right)
$$

Here we used notation $F^{\ddagger}=k_{\mathrm{B}} T / x^{\ddagger}$. This model predicts that in experiments performed with constantly ramped force the most probable rupture force $F^{*}$ at which $\mathrm{d} p_{\mathrm{BE}}(F) / \mathrm{d} F=0$ is proportional to the logarithm of the loading rate. Using $\varsigma=$ $F / F^{\ddagger}+\log \left(k_{0} F^{\ddagger} / v_{\mathrm{F}}\right)$, the PDF from eq 4 becomes $p_{\mathrm{BE}}(\varsigma)=\exp (\varsigma$ $-\exp (\varsigma)+1)$. Therefore, for different loading rates, the PDF shifts along the force coordinate, preserving the shape of the distribution even at the arbitrarily high rupture forces. This prediction is a consequence of assumptions used in this model: $\mathrm{d} v_{\mathrm{F}} / \mathrm{d} F=0$ and $\mathrm{d} x^{\ddagger} / \mathrm{d} F=0$. Models that do not assume stationary position of equilibrium and/or transition state predict that shape of the PDF (and consequently variance of rupture forces) changes for different loading rates. This change is a consequence of decreasing the barrier width with increasing force. ${ }^{5}$ Typical examples are shown in Figure 1. Here we compare probability distributions calculated according to the Bell-Evans model, PDF for paraboloidal potential with the cusp-like barrier (henceforth called "cusp model"), and PDF for the cubic-linear potential model., ${ }^{3,4}$ Parameters used in calculations (shown in the figure) are taken from our earlier work on rupturing biotin-streptavidin bonds. ${ }^{29}$ Loading rate in these calculations is given by the product of the probe velocity by the spring constant of the force sensor $v_{\mathrm{F}}=k_{\mathrm{s}} v$.

The model by Dudko et al. is another model that is often used in the analysis of force spectroscopy experiments. ${ }^{8}$ This model is based on Kramers' reaction rate theory and consequently is valid in the assumption of the high free energy barrier. From the dissociation rate dependence on the pulling force derived in this model, it can be concluded that there is a force $F_{\mathrm{mr}}$ where the dissociation rate $k(F)$ reaches the maximum $k_{\max }$ : 


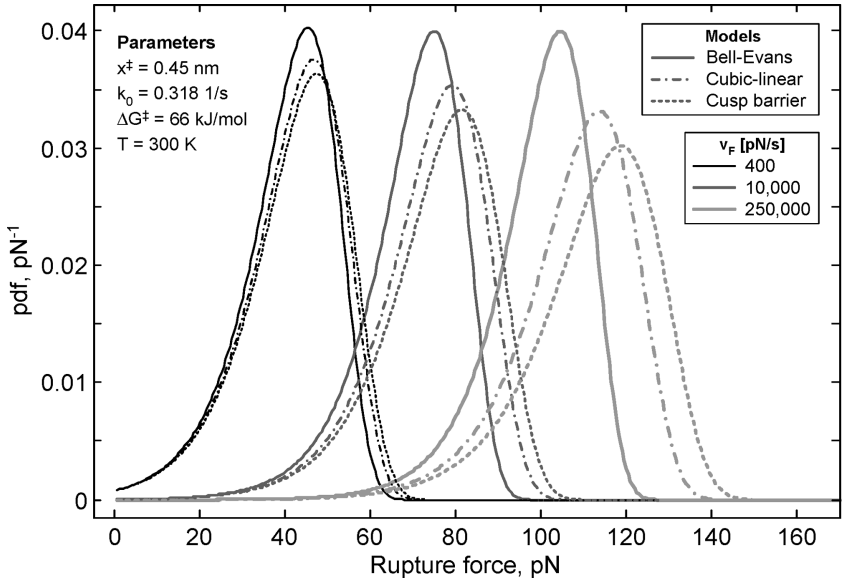

Figure 1. PDF for three different models. Calculation parameters are shown in the figure.

$$
\begin{gathered}
F_{\mathrm{mr}}=\frac{\Delta G^{\ddagger}}{\vartheta x^{\ddagger}}\left(1-\left(\frac{(1-\vartheta) k_{\mathrm{B}} T}{\Delta G^{\ddagger}}\right)\right) \\
k_{\max }=A e^{\vartheta-1}\left(\frac{(1-\vartheta) k_{B} T}{\Delta G^{\ddagger}}\right)^{1-\vartheta}
\end{gathered}
$$

Here $x^{\ddagger}$ is the barrier width at zero force, $\Delta G^{\ddagger}$ is the free energy difference between the equilibrium and transition state at zero force, $\vartheta$ is a characteristic exponent that equals to $1 / 2$ for the cusp model and to $2 / 3$ for the cubic-linear potential model, and $A$ is the Arrhenius pre-exponential factor such that $k_{0}=A$ $\exp \left(-\Delta G^{\ddagger} / k_{\mathrm{B}} T\right)$. Another characteristic force in this model is a critical force, $F_{\mathrm{cr}}=\Delta G^{\ddagger} /\left(\vartheta x^{\ddagger}\right)$, at which the barrier disappears and the dissociation rate decreases to zero. Decrease in the dissociation rate from the maximum value to zero in this model is a hallmark of the breakdown of the high-barrier approximation: at $F=F_{\mathrm{mr}}$, the free energy difference between the transition state and the ground state becomes $1 / 2 k_{\mathrm{B}} T$ for the cusp model and $1 / 3 k_{\mathrm{B}} T$ for the cubic-linear potential model. It has been suggested ${ }^{9}$ that for bonds that can survive high forces approaching $F_{\mathrm{cr}}$ the dissociation rate becomes constant. We note that if for forces exceeding $F_{\mathrm{mr}}$ the dissociation rate is fixed to the $k_{\max }$ value then survival probability calculated by eq 2 remains continuous $^{4}$ at $F>F_{\text {cr }}$. A consequence of this constant $k_{\max }$ assumption is that, when the mean rupture force approaches $F_{\text {mr }}$, a pronounced tail of high rupture forces appears in the distribution. This is illustrated in Figure 2 for two models of the free energy potential. These graphs show that the predicted appearance of the high force tail is sensitive to the loading rate: at lower loading rates, distributions are skewed to the lower forces (as in the Bell-Evans model), while for high loading rates, the distributions become skewed toward high forces. Therefore, it might be expected that if the tail of high rupture forces arises because of kinetic effects described here its amplitude should increase by increasing pulling velocity.

Next we consider conditions when such a high force tail can be measured. Here we limit our consideration to the cusp potential model. For this potential $(\vartheta=1 / 2)$ and constant $v_{\mathrm{F}}$, the bond survival probability is

$$
\begin{aligned}
& S(F)= \\
& \exp \left(-\frac{A k_{\mathrm{B}} T}{v_{\mathrm{F}} x^{\ddagger}}\left[\exp \left(-\frac{\left(F x^{\ddagger}-2 \Delta G^{\ddagger}\right)^{2}}{4 \Delta G^{\ddagger} k_{\mathrm{B}} T}\right)-\exp \left(-\frac{\Delta G^{\ddagger}}{k_{\mathrm{B}} T}\right)\right]\right)
\end{aligned}
$$

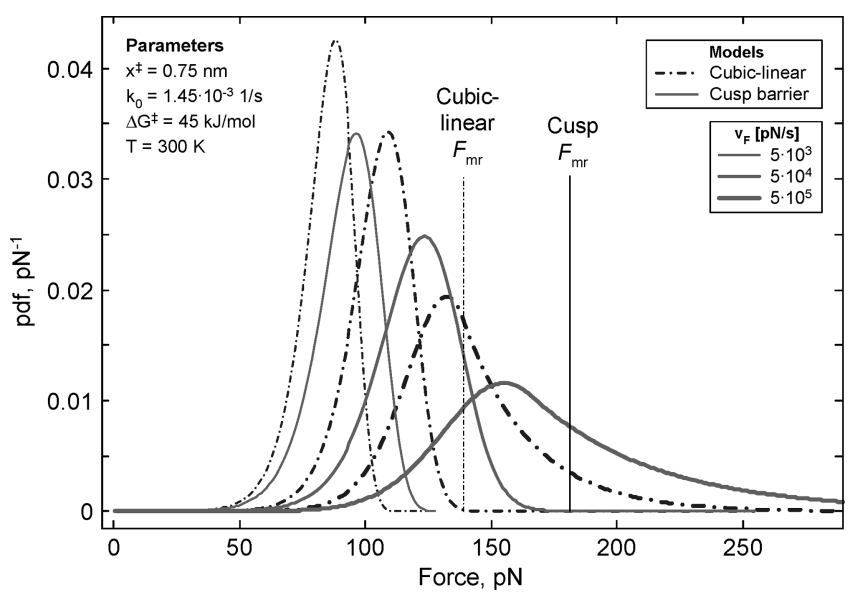

Figure 2. Appearance of the high force tail in the distributions of rupture forces. Vertical lines show positions of $F_{\mathrm{mr}}$ forces for each model of potential. Calculation parameters are shown in the figure.

For a bond to survive the increasing force up to $F_{\mathrm{mr}}$ with some minimum probability necessary for reliable detection of ruptures $S_{\min }$, the maximum pre-exponential factor is

$$
A_{\max } \approx v_{\mathrm{F}} x^{\ddagger} \exp (1 / 2) \log \left(1 / S_{\min }\right) / k_{\mathrm{B}} T
$$

If we suggest that to detect a tail of high forces the survival probability at force $F_{\mathrm{mr}}$ should exceed $\sim 0.1$ and if we consider a barrier with relatively large width $x^{\ddagger}$ of $1 \mathrm{~nm}$, then at room temperature numerically $A_{\max } \approx v_{\mathrm{F}}$ when the loading rate is expressed in $\mathrm{pN} / \mathrm{s}$ units. In AFM experiments, the loading rate usually does not exceed $10^{6} \mathrm{pN} / \mathrm{s}$. Therefore, a tail of high rupture forces might be expected only for molecular bonds with rather low pre-exponential factor $A<10^{6} \mathrm{~s}^{-1}$. We note that for the biotin-streptavidin bond the pre-exponential factor is considered to be in the range $\sim 10^{9}-10^{11} \mathrm{~s}^{-1} \cdot{ }^{30,32}$ Therefore, we expect that there should be no high force tail in the rupture of a single biotin-streptavidin bond in AFM-based experiments and in experiments that employ even lower loading rates (like optical tweezers, magnetic tweezers, or biomembrane force probe techniques).

For the cusp potential model, the pre-exponential factor equals ${ }^{4} A=(2 \pi)^{-1 / 2} D x^{\ddagger}\left(k_{\mathrm{m}} / k_{\mathrm{B}} T\right)^{3 / 2}$, where $D$ is the diffusion coefficient and $k_{\mathrm{m}}$ is the spring constant of a molecular bond. Therefore, detection of a high force tail is possible only if the diffusion coefficient is lower than the maximum value of

$$
D_{\max } \approx\left(2 \pi k_{\mathrm{B}} T \exp (1)\right)^{1 / 2} v_{\mathrm{F}} \log \left(1 / S_{\min }\right) / k_{\mathrm{m}}^{3 / 2}
$$

For a rather soft molecular bond with $k_{\mathrm{m}}=250 \mathrm{pN} / \mathrm{nm}$ and the same values for $v_{\mathrm{F}}$ and $S_{\min }$ as above, eq 9 gives $D_{\max } \approx 5$ $\times 10^{-15} \mathrm{~m}^{2} / \mathrm{s}$ (note that according to simulations and experimental measurements ${ }^{1,29}$ the biotin-streptavidin bond has $k_{\mathrm{m}}$ of $\sim 800 \mathrm{pN} / \mathrm{nm}$, and thus for this bond, $D_{\max } \sim 10^{-15} \mathrm{~m}^{2} / \mathrm{s}$ ). This diffusion coefficient is considerably lower than the diffusion coefficient of small molecules in an aqueous environment $\left(\sim 10^{-10} \mathrm{~m}^{2} / \mathrm{s}\right){ }^{36}$ Therefore, diffusion of a bound ligand should be significantly hindered in order for a high force tail to appear in the distribution of rupture forces.

Another factor that might widen distribution of rupture forces is a variation in the optical lever sensitivity (OLS) of an AFM instrument during the experiment because this sensitivity is used to convert measured optical signal into deflection of the force 
sensor. Our earlier measurements have indicated that OLS measured during force mapping experiments might vary by approximately $\pm 5 \% .{ }^{37}$ Consequently, the measured rupture forces vary by the same amount. This amount can be compared to the expected variance of measured rupture forces. ${ }^{8}$ Here we calculate variance using the Bell-Evans model for simplicity $(\vartheta=1)$. Then the width of the distribution of rupture forces is $\pi F^{\ddagger} / \sqrt{ } 6 .{ }^{8}$ Therefore, for $x^{\ddagger}=0.5 \mathrm{~nm}$, the expected width of the distribution at room temperature is $\sim 10 \mathrm{pN}$. Widening of the force distribution because of the sensitivity variation will be equal to this value for average rupture forces of $\sim 200 \mathrm{pN}$. This means that variation in sensitivity might be an important factor affecting width of the rupture force distribution, particularly for distributions with high most probable rupture forces.

2.2. Distribution of Rupture Forces in Single-Molecule Measurements with Anharmonic Tethers. When force is applied to a molecular bond by a flexible anharmonic tether that is pulled with constant velocity, the instantaneous loading rate depends on physical properties of tethers and conditions of experiment. Previously, we have considered tether effects in relation to the Bell-Evans model of potential. ${ }^{34,38}$ Here we briefly compare tether effects in relation to other models of PMF.

The loading rate as a function of force applied to a polymeric tether can be calculated by ${ }^{34}$

$$
v_{\mathrm{F}}(F)=\frac{v k_{\mathrm{c}}}{1+k_{\mathrm{c}} l^{\prime}(F)}
$$

Here $v$ is the probe velocity, $k_{\mathrm{c}}$ is the spring constant of the cantilever sensor, and $l(F)$ is the force-dependent length of a linker; the prime denotes differentiation with respect to force. The freely jointed chain (FJC) model is often used to describe pulling on polymeric tethers. ${ }^{33}$ Other frequently used models are the worm-like chain model and models that consider conformational transitions in polymeric chains under force. ${ }^{33,39}$ With the appropriate tether model for $l(F)$, eq 10 can be substituted into eqs 1 and 2 to calculate the expected PDF.

Incorporation of the tether between the bond and the force sensor decreases the loading rate from the nominal $v k_{\mathrm{c}}$ value as shown by eq 10 . When pulling is performed at constant velocity, the increase in the tether length results in the decrease of the most probable rupture forces (MPF), as shown in Figure 3A. Here calculations are performed using the FJC tether mode ${ }^{40}$ with the same kinetic parameters as in Figure 1. Figure 3B shows how the shape of the PDF for the Bell-Evans and the cusp potential models changes for tethers of different contour length. To perform such comparison, the pulling velocity was adjusted for each curve to keep the same MPF, as indicated in the legend. From these plots, it can be noted that relative widths of PDF increase with increasing tether length.

For polydisperse tethers, the resulting PDF is a weighted sum of distributions from tethers of different length. For the Bell-Evans model, we have shown previously that, if the distribution of tether lengths is not very wide and symmetric, the resulting PDF is nearly indistinguishable from the PDF calculated for the most probable tether length. ${ }^{34} \mathrm{We}$ have performed similar calculations for the cusp and cubic-linear potential models that also show that distributions of rupture forces for polydisperse and monodisperse tethers are very similar.

2.3. Effects of Pulling Geometry on Measured Parameters. 2.3.1. Distribution of Rupture Forces. Above we have considered the "ideal" case when the detected force is aligned
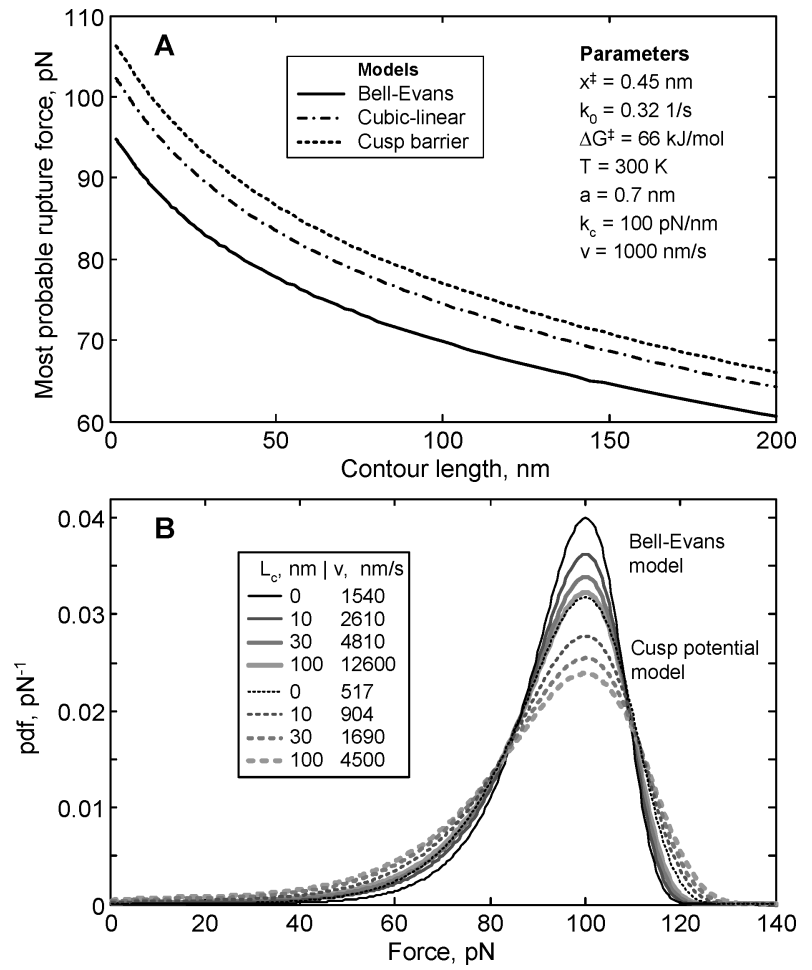

Figure 3. (A) Dependence of the most probable rupture forces (MPF) on tether length for three models. Parameters are shown in the graph. (B) Increase of the PDF width for longer tethers for the Bell-Evans and the cusp potential models. Pulling velocities are adjusted to keep the same MPF.
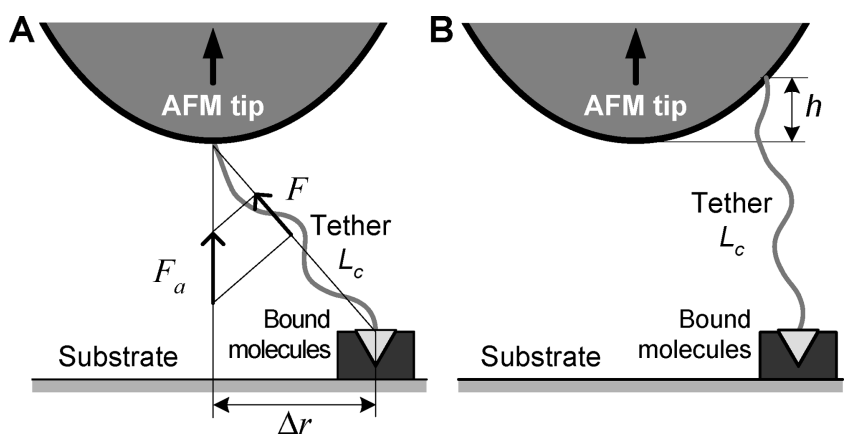

Figure 4. (A) Off-normal pulling geometry. (B) Geometry of the offapex tether attachment.

with the force acting on the bond. However, it has been shown that deviation of the pulling direction from the normal direction to the surface induces systematic error in the measured forces of molecular events. ${ }^{41,42}$ The off-normal pulling geometry is illustrated in Figure 4A. The apparent rupture forces detected in such geometry are expected to be higher than in the ideal geometry. ${ }^{41,42}$ The average PDF that includes effect of the offnormal pulling as a function of an apparent rupture force $F_{\mathrm{a}}$ can be calculated as

$$
p_{\mathrm{a}}\left(F_{\mathrm{a}}\right) \approx \int_{0}^{\infty} \mathrm{d} \Delta r p\left(F_{\mathrm{a}} \sqrt{1-\left(\Delta r / L_{\mathrm{c}}\right)^{2}}\right) p_{\mathrm{r}}(\Delta r)
$$

Here we integrate over all possible lateral offsets and assume that rupture occurs when the tether is stretched at an extension that is close to the tether contour length $L_{\mathrm{c}}$; factor $(1-(\Delta r)$ $\left.\left.L_{\mathrm{c}}\right)^{2}\right)^{1 / 2}$ is included to represent projection of the apparent force onto direction of a pulling tether (see Figure $4 \mathrm{~A}$ ), and $p_{\mathrm{r}}(\Delta r)$ is the probability density to find a particular lateral offset $\Delta r$. 
Assuming that the binding occurs at conditions when the tether can be described by a Gaussian chain model, $p_{\mathrm{r}}(\Delta r)$ can be estimated by

$$
p_{\mathrm{r}}(\Delta r)=\frac{3 \Delta r}{\left\langle r^{2}\right\rangle} \exp \left(-3 \Delta r^{2} / 2\left\langle r^{2}\right\rangle\right)
$$

where $\left\langle r^{2}\right\rangle=a L_{\mathrm{c}}$ is the mean square displacement of the unperturbed chain with the Kuhn length $a$. The off-normal pulling shifts the PDF toward higher force values and broadens the distribution. The apparent most probable rupture force can be estimated by $F_{\mathrm{a}}^{*}=F^{*}\left(1-\left(\langle\Delta r\rangle / L_{\mathrm{c}}\right)^{2}\right)^{-1 / 2}$. Square of the mean lateral offset is $\langle\Delta r\rangle^{2}=\pi\left\langle r^{2}\right\rangle / 6$, and therefore, $F_{a}^{*}=F^{*}(1-$ $\left.\pi /\left(6 N_{\mathrm{K}}\right)\right)^{-1 / 2}$, where $N_{\mathrm{K}}$ is the number of Kuhn segments in the tether. Therefore, shift of the PDF along the force axis $\left(\Delta F^{*}=\right.$ $\left.F_{a}^{*}-F^{*}\right)$ is $\Delta F^{*} \approx \pi F^{*} /\left(12 N_{\mathrm{K}}\right)$. Variance of the $\Delta r$ distribution is $\left\langle\Delta r^{2}\right\rangle-\langle\Delta r\rangle^{2}=(4-\pi)\left\langle r^{2}\right\rangle / 6$. Therefore, the PDF shift and broadening because of the off-normal pulling are small for tethers with lengths that are typically used in experiments $\left(N_{\mathrm{K}}\right.$ $>20$ ). Here we have neglected the possible effect that during the tip-sample contact the tether attached to the probe apex will be "squeezed out" from the contact area and therefore the lateral offset $\Delta r$ might be larger than predicted by the Gaussian chain model. We note that the attachment of tethers exactly at the apex is unlikely. Therefore, the off-normal pulling effects on distributions of rupture forces are expected to be small.

Another possible effect of pulling geometry is the off-apex tether attachment, as illustrated in Figure 4B. We do not expect that such off-apex attachment noticeably affects distribution of rupture forces. However, this effect is likely to affect distributions of other parameters, and these effects are considered next.

2.3.2. Distribution of Contour Lengths. Distributions of contour lengths of polymeric tethers in the bond dissociation experiments are often used to support the specific nature of measured interactions. ${ }^{43,44}$ Also, deviation of apparent contour length from the true value might affect values of the measured Kuhn length (as explained below). Therefore, in this section, we consider the likely sources of systematic and random errors in the contour length. If rupture forces are detected by pulling a single ligand that is tethered at the apex of the probe, the extracted distributions of contour and Kuhn lengths are expected to be narrow. For tethers attached close to the apex, the distribution of contour length extracted from AFM pulling experiments is expected to be similar to the distribution of tether lengths obtained from mass spectrometry measurements of molecular weights of tethers used in the tethering reaction. Mass spectrometry measurements of tethers used in this work give $L_{\mathrm{c}}=30 \pm 6 \mathrm{~nm}$, where error corresponds to two standard deviations of the width of the distribution. ${ }^{44}$ Another possible effect is that the size of surface-immobilized receptors also contributes to the extracted contour lengths. For example, size of streptavidin is $\sim 4.5 \mathrm{~nm}$, and therefore, this length might contribute to the extracted $L_{\mathrm{c}}$ if position of the probe-sample contact is measured at the solid substrate surface. It might be expected that for sparsely grafted molecules this contribution will be more noticeable for sharper tips that have higher probability to contact the substrate without compressing streptavidin molecules.

If the tether is attached off-apex, the apparent contour length will be shorter by the $z$ displacement of the attachment point $h$ (see Figure 4B). The tethered ligand should be able to reach the receptor during the probe-sample contact for a molecular bond to form. Therefore, for the AFM tips of paraboloidal shape that have radius of the apex curvature $R$ and the tethers with the mean square end-to-end displacement $\left\langle r^{2}\right\rangle$, the average offset $\langle h\rangle$ of the polymeric tether from the apex can be estimated by ${ }^{45}$

$$
\langle h\rangle=\frac{\int_{0}^{\infty} h \exp \left(-3 h^{2} / 2\left\langle r^{2}\right\rangle\right) \sqrt{R+2 h} \mathrm{~d} h}{\int_{0}^{\infty} \exp \left(-3 h^{2} / 2\left\langle r^{2}\right\rangle\right) \sqrt{R+2 h} \mathrm{~d} h}
$$

Here we assumed that the tether can attach anywhere on the probe's surface with equal probability, and we used the surface area element for a paraboloidal tip $\mathrm{d} S=2 \pi \sqrt{ } R(R+2 h) \mathrm{d} h$. Numerical estimates using contour lengths of tethers and probe sizes that are typically used in AFM experiments show that $\langle h\rangle$ $\approx 0.5\left\langle r^{2}\right\rangle^{1 / 2}$, and it is nearly independent of the probe radius (in the $R$ range from 5 to $100 \mathrm{~nm}$ ). Also, numerical calculations show that the variance of the offset $h$ is approximately $\left\langle h^{2}\right\rangle-$ $\langle h\rangle^{2} \approx 0.12\left\langle r^{2}\right\rangle$. Therefore, the off-apex tether attachment will widen the tether contour length distribution toward lower values by $\sim 1.2\left\langle r^{2}\right\rangle^{1 / 2}$.

Considering all of these sources of systematic errors, we expect that the distribution of tether contour lengths measured in our experiments will range approximately from 15 to $40 \mathrm{~nm}$. Here we have neglected the random errors in estimated contour lengths that might arise when fitting data contaminated by noise.

2.3.3. Distribution of Kuhn Lengths. It might be expected that in experiments with idealized geometry the extracted Kuhn length can be used as an indicator of a number of tethers that are being stretched in parallel. ${ }^{23,46}$ Therefore, in this section, we consider how various factors might affect the measured Kuhn lengths. The Kuhn length distribution might be expected to be narrow for tethers attached to the probe's apex because above (in section 2.3.1) we have shown that possible lateral offset between the attachment points will produce rather small variation in the pulling forces. Variation in characteristic ratio for tethers of different sizes might be expected to increase the width of the distribution of extracted Kuhn lengths. ${ }^{47}$ However, for tethers with relatively small polydispersity, this is unlikely to be a major effect. ${ }^{48}$ Next we consider how the systematic error in the extracted Kuhn length depends on the offset of tether attachment.

For a fixed value of tip-sample separation, the stretching force will increase with increasing the off-apex offset $h$. Therefore, the apparent Kuhn length will decrease with increasing $h$. On the other hand, surface roughness and the finite size of the receptor molecules might produce negative values of the offset $h$. Consequently, the apparent value of the Kuhn length will be higher. At high rupture forces, the relative error in the Kuhn length is $\delta a / a \approx-h / L_{\mathrm{c}}$. However, the detailed analysis (see the Supporting Information) shows that for low rupture forces the relative error in the Kuhn length can be higher than the high force limit by a factor of $\sim 1.5$. Therefore, for tethers used in this work, the Kunh length will be underestimated by up to $\sim 30 \%$ because of the tether off-apex attachment and overestimated by up to $\sim 30 \%$ because of the finite size of receptor molecules.

Errors in estimating the baseline force will also contribute to widening the Kuhn length distribution. If the baseline is estimated with $F_{\text {off }}$ error and assuming that all values of tip-sample separation $s$ are uniformly spaced, then the relative error in the Kuhn length determined from the least-squares fit of the FJC model will be $\delta a / a \approx-F_{\text {off }} \ln \left(F / F_{\mathrm{K}}\right) /\left(F-F_{\mathrm{K}}\right)$, where $F_{\mathrm{K}}=k_{\mathrm{B}} T / a$ is the characteristic thermal force of a polymeric chain and $F$ is the maximum stretching force (see the Supporting Information for derivations). For rupture forces of $\sim 10 F_{K}$, this 

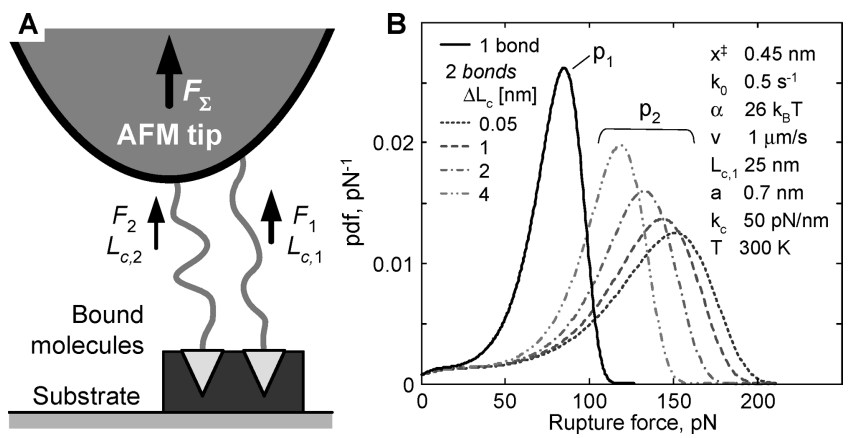

Figure 5. (A) Two-bond rupture geometry. (B) Probability of rupture forces for one- and two-bond ruptures when two tethers have different lengths. Parabolic potential with the cusp barrier was used in the kinetic model, and the eFJC model was used to calculate dynamics of loading. The legend and calculation parameters are shown in the graph.

error is approximately $-2 F_{\text {off }} / F$. For typical $F_{\mathrm{K}}=6 \mathrm{pN}$, the baseline offset of $\pm 2 \mathrm{pN}$ will result in approximately $\sim 7 \%$ error in the Kuhn length. In experimental data, the force baseline sometimes experiences slow drift. Therefore, to minimize this source of error in the extracted Kuhn length, the baseline should be corrected at the force plot position immediately after the rupture event. If the data are collected with the $\sigma_{\mathrm{F}}$ rms force noise and with the uniform separation $\lambda$ between the data points, then the random error in the baseline position estimated over distance $x$ is $\left(\sigma_{\mathrm{F}}^{2} \lambda / x\right)^{1 / 2}$, and the systematic error in the baseline force is $b x / 2$, where $b$ is the slope of the linear baseline drift. Therefore, the optimum distance to correct for the baseline noise that minimizes the total error is $x^{*}=\left(\lambda^{1 / 2} \sigma_{\mathrm{F}} / b\right)^{2 / 3}$. This expression expectantly predicts that if there is no baseline drift then all data collected after the rupture should be used for baseline correction. In experimental data, some drift is usually present; moreover, the baseline drift might be not constant, for example, because of the optical interference artifacts. Therefore, we usually correct the baseline by averaging data collected over $30 \mathrm{~nm}$ distance after the rupture. Consequently, the baseline error is $\left(\sigma_{\mathrm{F}}^{2} \lambda / x+\sigma_{\mathrm{b}}^{2}(x / 2)^{2}\right)^{1 / 2}$, where $\sigma_{\mathrm{b}}$ is the rms of the baseline slope. For the data that we collected, typical baseline offset error from the random noise is $1-2 \mathrm{pN}$ and from the baseline slope is $3-4.5 \mathrm{pN}$. Therefore, total baseline error ranges from 2 to 5 $\mathrm{pN}$. Corresponding variation in the Kuhn length extracted from rupturing single bonds is expected to reside within approximately $\pm 15 \%$ range of the mean value. This error is considerably lower than the error arising from the off-apex attachment. The overall error in the Kuhn length is expected to be $\pm 35 \%$ of the mean value. In this estimate, we have neglected effects of random errors in the force data that might further increase the width of the measured Kuhn length distribution.

2.4. Effects of Simultaneous Rupture of Two Bonds on Measured Parameters. When the AFM probe is connected to the substrate by more than one bond, sequential rupture of these bonds can be considered as a series of independent ruptures. If two sequential ruptures occur nearly simultaneously, then only one rupture event will be detected. ${ }^{49}$ We have considered this effect earlier ${ }^{16,29}$ and found that, when tethers for two bonds have different contour length, the apparent rupture force might have a pronounced tail on a high force side of the distribution. This effect might be expected to be common when rupture from multivalent receptors is been investigated (Figure 5A). Here we briefly consider the distribution of rupture forces when two independent bonds are loaded and ruptured nearly simultaneously and also consider how stretching of two parallel tethers of different length affects the extracted Kuhn lengths.
2.4.1. Distribution of Rupture Forces. Consider two tethers that are stretched in parallel (Figure 5A). Then the detected force is the sum of forces on individual chains: $F=F_{1}+F_{2}$. If two independent bonds rupture nearly simultaneously and if such ruptures are detected as one rupture event, then the total survival probability $S$ approximately equals the product of individual survival probabilities: ${ }^{16}$

$$
S(F) \approx s_{1}\left(F_{1}\right) s_{2}\left(F_{2}\right)
$$

If two bonds are governed by identical energy landscapes, then this equation can be written as

$$
S(F) \approx s\left(F_{1}\left(F, L_{\mathrm{c}}, \Delta L_{\mathrm{c}}\right)\right) s\left(F-F_{1}\left(F, L_{\mathrm{c}}, \Delta L_{\mathrm{c}}\right)\right)
$$

Here $s(F)$ is the survival probability of one bond, $L_{\mathrm{c}}$ is the contour length of one tether, and the contour length of the second tether is $L_{\mathrm{c}, 2}=L_{\mathrm{c}}+\Delta L_{\mathrm{c}}$. If two tethers have the same length, then $F_{1}=F / 2$ and $S(F)=\mathrm{s}^{2}(F / 2)$. Consequently, the total PDF function is $P(F)=\mathrm{s}(F / 2) p(F / 2)$. One consequence of this equation is that the most probable force for rupture of two identical bonds in parallel is less than $2 F^{*}$ because $s(F)$ is a monotonically decreasing function of force. For tethers with different length, the most probable rupture force is even lower because the bond connected by the shorter tether will experience higher force and will rupture first with higher probability. ${ }^{16}$ Consequently, if the measured rupture events contain contributions from one-bond and two-bond ruptures, the two peaks in the distribution are likely to merge into a single peak with a width considerably exceeding the width of a distribution for single-bond ruptures. ${ }^{29}$

2.4.2. Distribution of Rupture Forces and Binding Probability. In a previous article, we showed that for a tethered ligand that diffuses in solution the probability to bind to a surfaceimmobilized receptor $P_{\text {bind }}$ depends on time that the ligand spends in the vicinity of the receptor. ${ }^{45}$ If the receptor is multivalent and there are several ligands tethered on the probe, then the probability that two bonds will be formed might be considerable. Therefore, the number of multiple bond ruptures is expected to decrease in experiments with decreasing probe-sample contact time. The cumulative PDF of rupture forces can be calculated by ${ }^{29}$

$$
p_{\mathrm{tot}}(F)=\sum_{j} f_{j} p_{j}(F)
$$

Here $p_{j}$ is the PDF for simultaneous rupture of $j$ bonds and $f_{j}$ is a fraction of $j$ bond ruptures in the total distribution. Fractions $f_{j}$ depend on many experimental parameters such as number and mobility of ligands, distribution and valence of receptors, probe velocity, and the time that the probe rests on the surface, surface elasticity, and other factors. Examples of functions $p_{1}$ and $p_{2}$ when the number of simultaneously ruptured bonds does not exceed two and when two tethers have different lengths are shown in Figure 5B. If fractions $f_{j}$ of multiple bonds $(j>1)$ are not small at different probe velocities, then distributions of rupture forces will have a noticeable tail of high forces that might have velocity dependence that is different from the velocity dependence of high forces arising from the single-bond rupture kinetics (see section 2.1). This effect is considered next. 


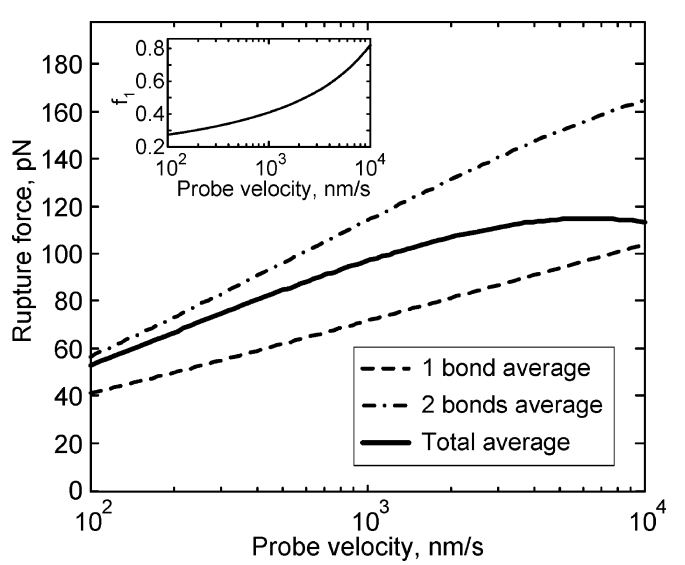

Figure 6. Dependence of average rupture forces on pulling velocity for divalent receptor and two ligands tethered by separate chains at the probe apex. The inset graph shows a fraction of single-bond occurrences in all binding events. Other parameters and the model are shown in the Supporting Information (see Figure S6).

Binding probability of a tethered ligand depends on probe velocity. ${ }^{45}$ Therefore, it might be expected that probability to form multiple bonds also depends on probe velocity. For simplicity, we have considered binding of one and two ligands to a divalent receptor. Considering independent binding of two ligands to one receptor, probabilities to form one and two bonds ( $P_{1}$ and $P_{2}$, respectively) can be calculated by

$$
\begin{gathered}
P_{1}=\frac{2}{3} \pi \theta\left\langle r^{2}\right\rangle \log \left(N_{\operatorname{lig}} \log \left(1 / \tilde{s}_{2}\right) / \log (2)\right) \\
P_{2}=\frac{2}{3} \pi \theta\left\langle r^{2}\right\rangle \log \left(A^{*}\left[t_{\text {dwell }}+\sqrt{2 \pi\left\langle r^{2}\right\rangle / 3} / v\right] / \log \left(1 / \tilde{s}_{2}\right)\right)
\end{gathered}
$$

Here $\theta$ is the surface density of divalent receptors, $\left\langle r^{2}\right\rangle$ is the mean square displacement of the unperturbed polymeric tether, $N_{\text {lig }}$ is a number of equivalent ligands tethered to the probe, $v$ is the probe velocity, $t_{\mathrm{dwell}}$ is the dwell time the probe spends in contact with the surface, $A *$ is the effective rate of receptor-ligand collisions resulting in bond formation, and $\tilde{s}_{2}$ is a real root in the range from 0 to 1 of equation $\tilde{s}_{2}^{N \text { lig }}-1\left(\tilde{s}_{2}^{N}\right.$ lig $\left.-\tilde{s}_{2}-1\right)+1 / 2=$ 0 . Derivation of eq 16 and further discussion is given in the Supporting Information. Fractions of one and two bonds that can be substituted in eq 15 are given by $f_{1}=P_{1} /\left(P_{1}+P_{2}\right)$ and $f_{2}=P_{2} /\left(P_{1}+P_{2}\right)$, respectively.

An example of expected change in the average rupture forces measured at different probe velocities is shown in Figure 6. Here the average force is bracketed by average forces of oneand two- bond ruptures; however, the slope does not remain constant in semilog coordinates. The major factor in the slope variation here is the difference in relative contributions of oneand two-bond ruptures as indicated by dependence of $f_{1}$ factor shown in the inset. The shown dependence indicates that probability of multiple bonds is decreasing with increasing probe velocity. This velocity dependence of the high force tail contrasts the velocity dependence shown in Figure 2. Therefore, we suggest that velocity dependence of the probability of occurrence of high forces in the distribution of rupture forces might be used to elucidate nature of high rupture forces for soluble tether/ ligand system: If probability of high forces decreases with increased probe velocity, then high forces are likely originating from ruptures of multiple bonds. This suggestion is helpful for an explicit analysis of the PDF dependence on pulling velocity that is aimed at separating the single-bond ruptures from the multiple-bond ruptures and accurately estimating kinetic parameters of dissociation reaction. ${ }^{29}$

The above model (eq 16) considers binding of ligands to the same receptor. Consequently, fractions $f_{1}$ and $f_{2}$ are independent of grafting density. High fraction of multiple-bond ruptures (similar to the fraction obtained in calculations shown in Figure 6) originates from the multivalent nature of a receptor. (For high surface densities of receptors, the fraction of multiple bonds might be even higher because binding of different ligands to separate receptors might occur.) In contrast, the overall binding probability can be made low by reducing the receptor surface density. For example, for calculations shown in Figure 6, the overall binding probability will be $2-4 \%$ if surface density of receptors is $\sim 200 \mu \mathrm{m}^{-2}$. Therefore, low binding probability does not guarantee the single-bond nature of detected rupture events.

2.4.3. Distribution of the Contour Lengths and Kuhn Lengths. Using the high force asymptotic of the FJC model and assuming the same Kuhn length $a$ for both chains and also neglecting difference in the attachment points, we can write the total force sensed by AFM probe as ${ }^{16}$

$$
F \approx F_{\mathrm{K}}\left(\frac{1}{1-r}+\frac{1}{1-(r-\delta r)}\right)
$$

where $F_{\mathrm{K}}$ is the characteristic thermal force of polymeric tether, $F_{\mathrm{K}}=\left(k_{\mathrm{B}} T\right) / a, r=x / L_{\mathrm{c}}$ is the extension ratio of chain with the contour length $L_{\mathrm{c}}, x$ is the end-to-end distance, and $\delta r$ is the decrease in the extension ratio for the second (longer) chain: $\delta r \approx r \delta L_{\mathrm{c}}=r \Delta L_{\mathrm{c}} / L_{\mathrm{c}}, \Delta L_{\mathrm{c}}$ is the difference in the contour length of the longer and the shorter chains. In the data analysis, force curves are fit by a model that considers stretching of a single chain:

$$
F_{\mathrm{fit}}=\frac{k_{\mathrm{B}} T}{a_{\mathrm{app}}}\left(\frac{1}{1-x / L_{\mathrm{c}, \mathrm{app}}}\right)
$$

Here $a_{\text {app }}$ is the apparent Kuhn length and $L_{\mathrm{c} \text {,app }}$ is the apparent contour length. From eqs 17 and 18, it is obvious that if $\Delta L_{\mathrm{c}}=$ 0 then the apparent Kuhn length is $a / 2$. Numerical estimations using the least-squares approach (see the Supporting Information) show that even if the difference between contour lengths $\Delta L_{\mathrm{c}}$ is not negligible the apparent Kuhn length is higher than $a / 2$ by less than $3 \%$. Considering that the distribution of rupture forces is significantly affected by $\Delta L_{\mathrm{c}}$, this result is somewhat unexpected. We note that if in eq 18 we set $L_{\mathrm{c} \text {,app }}=L_{\mathrm{c}}$ then the apparent Kuhn length will deviate from $a / 2$ much more significantly (by up to 30\%). Therefore, explanation for $a_{\text {app }}$ being close to $a / 2$ is that the apparent contour length is becoming longer with increasing $\Delta L_{\mathrm{c}}$, and such an increase compensates for the increase in the apparent Kuhn length. Consequently, if random error in Kuhn length is not large, the distribution of Kuhn lengths is expected to be a robust indicator of multiplebond ruptures. Considering the discussion in section 2.3, we expect that this might be achieved when width of distribution of the extracted contour lengths does not significantly exceed the width of distribution of tethers measured in solution.

The apparent contour length will be in the range from $L_{\mathrm{c}}$ to $L_{\mathrm{c}}+\Delta L_{\mathrm{c}} / 2$ (see the Supporting Information). Therefore, ruptures of multiple bonds are not expected to widen distribution of the extracted contour lengths.

2.5. Numerical Simulation. Here we consider how the parameters extracted in one- and two-bond ruptures are affected by offset of the tether attachment point and by the finite size of 

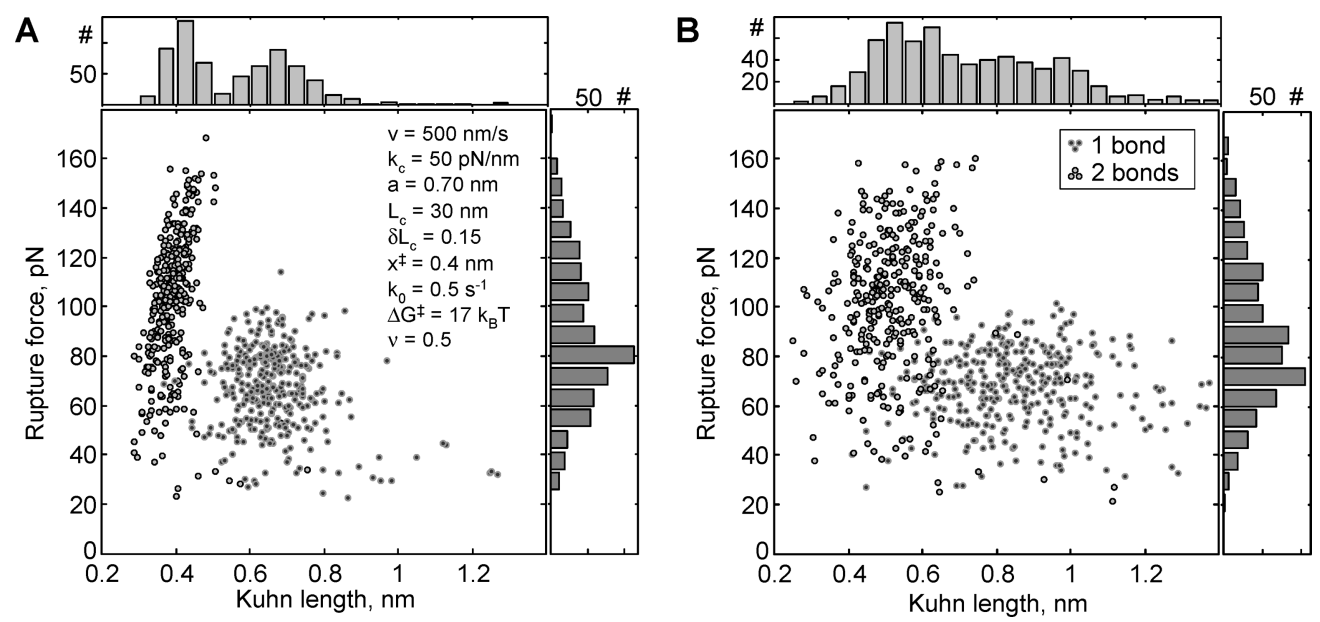

Figure 7. Rupture forces and Kuhn lengths extracted from simulations of one- and two-bond ruptures. (A) Results of calculations where the tethers are attached to the apex. (B) Results where the attachment point was randomly varied around $-4.5 \mathrm{~nm}$ with the standard deviation of $3 \mathrm{~nm}$. Subplots along the axes show the corresponding histograms of rupture forces and Kuhn lengths. Other parameters are shown in panel A, and the legend is shown in panel B.

a receptor. We have simulated force curves including the twobond rupture and the attachment offset effects in our calculations. The resulting force curves are analyzed using the same program that is used to analyze experimental data. The extracted rupture forces and Kuhn lengths are shown in Figure 7. Simulation procedure is described in the Supporting Information, where we also show the contour length distributions.

Results of calculations without the tether offset shown in panel A indicate that rupture forces from one and two bonds blend together into a wide distribution that does not permit clear separation between these ruptures. Distribution of Kuhn lengths is bimodal, supporting results of the analytical model from section 2.4.3. Results that include the systematic negative tether offset and random variation in tether attachment point are shown in panel B. It can be noticed that variation in the tether attachment does not significantly alter the distribution of rupture forces. Two peaks in the distribution of Kuhn lengths are now nearly merged together, illustrating the significant effect of the tether offset on the extracted Kuhn length, as discussed in section 2.3.3. It might be expected that this effect becomes even more pronounced for soft samples where binding events can occur with significantly larger off-apex offset than considered here.

\section{Materials and Methods}

3.1. Sample Preparation. All chemicals were purchased from Sigma-Aldrich, unless indicated otherwise. Samples including probes and glass substrates were prepared using the previously reported methods ${ }^{29,45}$ except for the sharp silicon probes (MikroMasch, San Jose, CA; model CSC38/AlBS) as described below. These silicon probes possess a typical radius of curvature of $\sim 10 \mathrm{~nm}$ and are considerably sharper than regular silicon nitride probes with a typical radius of curvature of 20-40 nm (Veeco, Santa Barbara, CA; model NP). For silicon probes with alumina coating on the back side, all sample preparation steps are the same, except that these probes are cleaned by being soaked in $2 \%$ Hellmanex II (Hellma, Müllheim, Germany) aqueous solution for $20 \mathrm{~min}$ instead of $3 \mathrm{~h}$ cleaning used for regular silicon nitride probes with gold coating on the back side. Poly(ethylene glycol) (PEG) tethers with average molecular mass of 3400 Da (NHS-PEG-Biotin, Laysan Bio Inc., Arab, AL) were used to covalently attach biotin ligands to the probes.

3.2. Data Collection and Analysis. Data collection procedures were similar to those previously reported. ${ }^{29,45}$ In experi- ments with different probe velocities, the data were collected by cycling probe velocities from high to low values and back several times in order to distribute effects of the tip wearing off over the entire data set. At least four force volumes (1024 force curves for each force volume) were collected for each probe velocity in all experiments. Zero and $0.2 \mathrm{~s}$ dwell time settings were used during the data collection. Data analysis procedures were also similar to those previously reported. ${ }^{29,45}$ Force curves containing probable rupture events were detected automatically using several criteria. Initial selection included identification of abrupt force transitions toward the baseline with amplitudes of the transitions equal to $6 \mathrm{rms}$ force noise value. Then transitions going to the baseline within $3 \mathrm{rms}$ force noise values and occurring at separations greater than $8 \mathrm{~nm}$ and lower than $60 \mathrm{~nm}$ were selected. The indicated range of tip-sample separations includes the expected range of polymeric tether stretching. The stretching part of selected force curves prior to the last rupture event have been fit by an extend FJC model ${ }^{50}$ to extract the contour lengths, the Kuhn length, and the rupture force. The whole selection procedures have been automatically conducted in order to avoid personal bias.

3.3. Confirmation of Interactions Specificity. Molecular interpretation of measured rupture forces requires that measured interactions correspond to ruptures of molecular bonds under study and not to spurious surface effects such as adhesion or contamination. Therefore, additional controls are necessary to establish the specific nature of measured interactions. We establish the specific nature of measured interactions using the "empty tether" approaches..$^{15,29,32,40,51-53}$ In empty tether control experiments, one of a pair of molecules involved in the measured interactions is removed, blocked, or chemically modified. In a control experiment with the AFM probe modified with PEG tethers without biotin, only 12 rupture events out of 6144 force curves $(0.2 \%$ detection probability) have been identified by our data analysis approach as rupture events. This detection probability is significantly less than that in the regular measurements $(\sim 3 \%)$ where PEG tethers are equipped with biotin. Other control experiments with free biotin in solution as described previously ${ }^{29}$ were conducted for each type of experiment, and we observe a significant decrease in binding probability similar to others. ${ }^{15,53}$ 


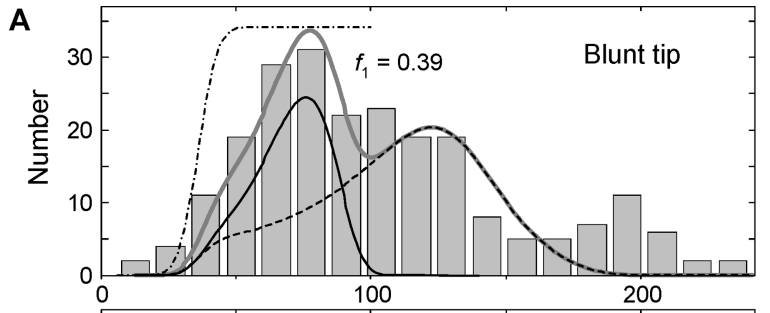

B
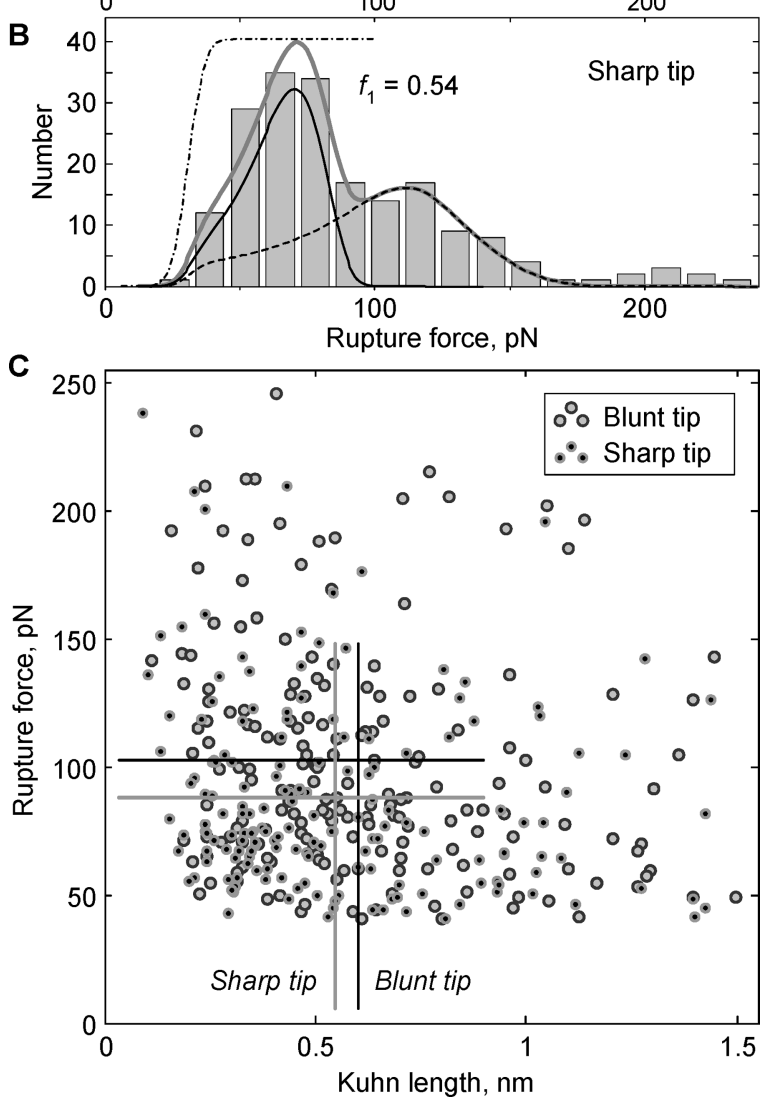

Figure 8. Histograms of rupture forces $(A, B)$ and scatter plots of rupture forces vs Kuhn lengths (C) extracted from measurements with a blunt and a sharp probe. Lines in panels A and B show fits by a two-bond rupture model; lines in panel $\mathrm{C}$ show mean values of parameters. Examples of force plots and individual histograms are shown in Figure S9 in the Supporting Information.

\section{Experimental Results}

4.1. Effects of Probe Size. Histograms of rupture forces and scatter plots of rupture forces versus Kuhn lengths from experiments using different probe sizes are shown in Figure 8. Lines in panels $\mathrm{A}$ and $\mathrm{B}$ are the fit lines according to the twobond rupture model. ${ }^{29}$ The only parameters that were different in fitting data from panels $\mathrm{A}$ and $\mathrm{B}$ by the two-bond rupture model are the amplitudes of the one- and two-bond rupture components; fractions of single-bond ruptures are shown in the corresponding panels. It is likely that a blunt tip has a larger number of biotin ligands tethered near the apex. Therefore, a fraction of single-bond ruptures is expected to be lower for blunt probes (see inset in Figure S6 in Supporting Information that supports this expectation). This expectation is consistent with differences between panels A and B. Scatter plots in panel C do not show well-separated clusters corresponding to ruptures of one and two bonds as in Figure 7A; instead, overall distribution is similar to the distribution in Figure 7B. Mean value of rupture force for the blunt tip is higher than the mean value for the sharp probe by $15 \mathrm{pN}$ (16\% difference). This difference might be obtained in single-molecule experiments if

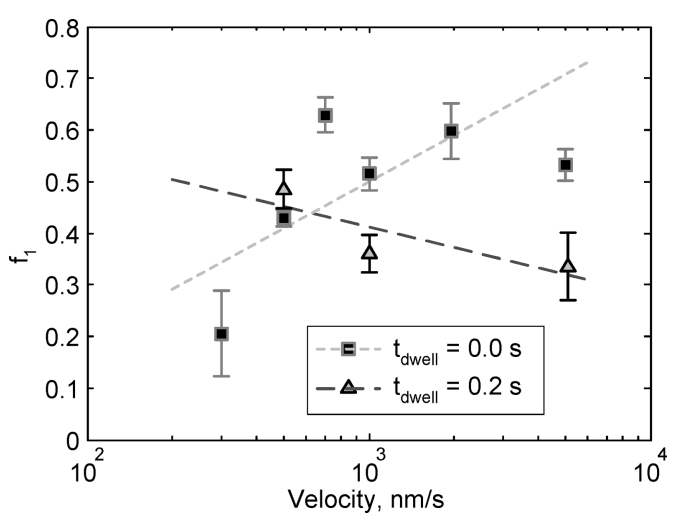

Figure 9. Relative amplitude of single-bond ruptures vs probe velocity for two dwell times. Dashed lines are the guides to the eye. The errors shown for each $f_{1}$ value were obtained by fitting histograms using different bin sizes ranging from 8 to $15 \mathrm{pN}$.

the pulling velocity is different by a factor of $\sim 5$, but in experiments shown here, it mainly comes from a higher fraction of the multiple-bond ruptures.

The mean Kuhn length for the blunt tip is higher than the mean Kuhn length for the sharp tip by $9 \%$. It might be expected that a higher fraction of multiple ruptures detected with the blunt tip generates lower mean Kuhn length. This expectation contradicts the observed difference. This contradiction is considered in the Discussion section below.

4.2. Probability of Multiple Bond Ruptures at Different Probe Velocities. We have measured the dependence of rupture force on probe velocity at two different values of the time the probe rests on the surface at the end of the approach part of the approach-withdraw cycle ("dwell time"). Measured histograms of rupture forces were fit simultaneously using the two-bond rupture model (see Figure S10 in the Supporting Information). ${ }^{29}$ Figure 9 shows the extracted dependencies of relative amplitude of a single-bond rupture on probe velocity. Amplitudes measured with $0.2 \mathrm{~s}$ dwell time do not show a significant variation for different probe velocities, while amplitudes measured with zero dwell time show noticeable increase in amplitudes with the probe velocity. The observed behavior is consistent with theoretical expectation (see section 2.4.2 above).

We note that at the largest probe velocity $(5 \mu \mathrm{m} / \mathrm{s})$ noise of the force data became substantial, and this might result in the systematic decrease of $f_{1}$ at these probe velocities. ${ }^{29}$ We suggest that this observation and also heterogeneity of the streptavidin surface coverage are two likely reasons for noticeable scatter in the graphs shown in Figure 9.

\section{Discussion}

The fraction of single-bond ruptures measured with zero dwell time increases with increasing probe velocity. This observation supports the earlier suggestion ${ }^{29}$ that high forces in the biotin-streptavidin distribution originate from simultaneous ruptures of multiple bonds. The ligands on the probe can reach the receptors on the surface and form bonds only when the probe is close to (much less than the tether stretched length) or contacts the substrate. In zero dwell time measurements, the probe velocity determines the duration of time within which the ligands can reach receptors on the substrate. However, in $0.2 \mathrm{~s}$ dwell time measurements, the dwell time is considerably longer compared to the probe travel time within which the ligand can reach the receptors on the substrate. Therefore, no significant variation in the fraction of single-bond ruptures is expected for different probe velocities in the measurements with $0.2 \mathrm{~s}$ dwell 
time. Presented model of relative probability of one- and twobond ruptures predicts that the fraction of single-bond ruptures should be considerably lower for data collected with nonzero dwell time than for data with zero dwell time. Although $f_{1}$ fractions for data with $0.2 \mathrm{~s}$ dwell time are lower than for data with zero dwell time, the measured difference is small (see Figure 9). One possible explanation why the measured difference is small is that the two-bond rupture model used in our data analysis does not account for all multiple rupture events and therefore might overestimate the $f_{1}$ fraction.

Measurements using relatively sharp probes indicate that with such probes probability of multiple rupture events is lower than with blunt probes. The Kuhn length of the tether measured using sharp probes was a bit lower than with blunt probes. This dependence contradicts the apparent decrease in number of multiple interactions measured with the sharp probes. However, with sharp probes, the number of tethers attached off-apex is relatively larger than with blunt probes. This increase in the attachment offset will decrease the apparent Kuhn length (see Figure S2 in the Supporting Information). This might explain the discrepancy between the average rupture forces and the average Kuhn length.

Measured distributions of contour lengths range from 10 to $50 \mathrm{~nm}$ (Figure S9). This range agrees reasonably well with the expected range from 15 to $40 \mathrm{~nm}$ (section 2.3.2). The somewhat narrower range of the expected contour length might have two contributions. One is that for ruptures that occur at the tether extensions that are noticeably less than the contour length, the random noise in the data might affect the extracted contour length values. Also some deviation of contour lengths from mass spectrometry results toward higher values is expected because of the preferential attachment of longer chains to the surface during sample prparation. ${ }^{54}$ Therefore, measured distribution of contour lengths might be somewhat wider than predicted above. Overall, the width of the measured distribution is considerably larger than the width expected from tether polydispersity. This indicates that effects of the off-apex attachment might be significant.

According to the theoretical models given above, the offapex tether attachment is a significant factor in widening distributions of extracted Kuhn lengths. Such widening might preclude from using Kuhn length as an indicator of multiplebond ruptures. Here we note that additional source of widening of the Kuhn length distribution is an experimental error in determining the surface position. On the other hand, rather precise measurements of Kuhn length can be achieved by stretching considerably longer polymeric molecules. ${ }^{55}$ Therefore, we expect that distribution of the Kuhn length in measurements with long tethers can be used to distinguish between single and multiple ruptures because effects of the off-apex attachment and uncertainty of the surface position will be relatively lower with long tethers than with short tethers. However, it has been suggested that there is the upper practical limit for the tether length in force spectroscopy measurements. ${ }^{56}$

\section{Conclusions}

In conclusion, we note that analytical models derived in this article explain distributions of parameters measured in force spectroscopy measurements reasonably well. However, predicted clustering of Kuhn lengths corresponding to one- and two-bond ruptures was not clearly demonstrated experimentally. Experimental verification of this prediction will require measurements with longer tethers or with ligand molecules tethered exclusively close to the probe apex.
Systematic variation in the shape of measured distributions of rupture forces supports the hypothesis of the multiple-bond nature of high rupture forces. We expect that the developed test can be applied to other receptor-ligand systems to examine whether measured distributions of rupture events have significant contribution from multiple-bond ruptures. Such measurements require that ligands are tethered by the relatively long soluble linkers and that ligands are not adsorbed to the surface of the probe.

Acknowledgment. The authors thank NSF Grant CHE0719043 for financial support.

Supporting Information Available: Details of the derivation for models described in the main text, description of the force plot simulation procedure, and histograms of the data. This material is available free of charge via the Internet at http:// pubs.acs.org.

\section{References and Notes}

(1) Evans, E.; Ritchie, K. Biophys. J. 1997, 72, 1541.

(2) Izrailev, S.; Stepaniants, S.; Balsera, M.; Oono, Y.; Schulten, K. Biophys. J. 1997, 72, 1568.

(3) Dudko, O. K.; Filippov, A. E.; Klafter, J.; Urbakh, M. Proc. Natl. Acad. Sci. U.S.A. 2003, 100, 11378.

(4) Hummer, G.; Szabo, A. Biophys. J. 2003, 85, 5.

(5) Bustamante, C.; Chemla, Y. R.; Forde, N. R.; Izhaky, D. Аnnu. Rev. Biochem. 2004, 73, 705 .

(6) Freund, L. B. Proc. Natl. Acad. Sci. U.S.A. 2009, 106, 8818.

(7) Evans, E. Faraday Discuss. 1998, 111, 1. 108101

(8) Dudko, O. K.; Hummer, G.; Szabo, A. Phys. Rev. Lett. 2006, 96,

(9) Sheng, Y. J.; Jiang, S. Y.; Tsao, H. K. J. Chem. Phys. 2005, 123, 061106.

(10) Hanke, F.; Kreuzer, H. J. Phys. Rev. E 2005, 72, 031805.

(11) Dudko, O. K.; Hummer, G.; Szabo, A. Proc. Natl. Acad. Sci. U.S.A. 2008, 105, 15755

(12) Serpe, M. J.; Kersey, F. R.; Whitehead, J. R.; Wilson, S. M.; Clark, R. L.; Craig, S. L. J. Phys. Chem. C 2008, 112, 19163.

(13) Oberbarnscheidt, L.; Janissen, R.; Oesterhelt, F. Biophys. J. 2009, 97, L19.

(14) Williams, P. M. Anal. Chim. Acta 2003, 479, 107.

(15) Sulchek, T. A.; Friddle, R. W.; Langry, K.; Lau, E. Y.; Albrecht, H.; Ratto, T. V.; DeNardo, S. J.; Colvin, M. E.; Noy, A. Proc. Natl. Acad. Sci. U.S.A. 2005, 102, 16638.

(16) Gu, C.; Kirkpatrick, A.; Ray, C.; Guo, S.; Akhremitchev, B. B. J. Phys. Chem. C 2008, 112, 5085.

(17) Rief, M.; Gautel, M.; Oesterhelt, F.; Fernandez, J. M.; Gaub, H. E. Science 1997, 276, 1109.

(18) Rief, M.; Pascual, J.; Saraste, M.; Gaub, H. E. J. Mol. Biol. 1999, $286,553$.

(19) Fisher, T. E.; Marszalek, P. E.; Fernandez, J. M. Nat. Struct. Biol. 2000, 7, 719 .

(20) Sharma, D.; Perisic, O.; Peng, Q.; Cao, Y.; Lam, C.; Lu, H.; Li, H. Proc. Natl. Acad. Sci. U.S.A. 2007, 104, 9278.

(21) Averett, L. E.; Geer, C. B.; Fuierer, R. R.; Akhremitchev, B. B.; Gorkun, O. V.; Schoenfisch, M. H. Langmuir 2008, 24, 4979.

(22) Averett, L. E.; Schoenfisch, M. H.; Akhremitchev, B. B.; Gorkun, O. V. Biophys. J. 2009, 97, 2820.

(23) Sarkar, A.; Caamano, S.; Fernandez, J. M. Biophys. J. 2007, 92, L36.

(24) Florin, E. L.; Moy, V. T.; Gaub, H. E. Science 1994, 264, 415.

(25) Lo, Y. S.; Huefner, N. D.; Chan, W. S.; Stevens, F.; Harris, J. M.;

Beebe, T. P. Langmuir 1999, 15, 1373.

(26) Seifert, U. Phys. Rev. Lett. 2000, 84, 2750.

(27) Tees, D. F. J.; Woodward, J. T.; Hammer, D. A. J. Chem. Phys. 2001, 114, 7483

(28) Ratto, T. V.; Rudd, R. E.; Langry, K. C.; Balhorn, R. L.; McElfresh, M. W. Langmuir 2006, 22, 1749.

(29) Guo, S.; Ray, C.; Kirkpatrick, A.; Lad, N.; Akhremitchev, B. B. Biophys. J. 2008, 95, 3964.

(30) Pincet, F.; Husson, J. Biophys. J. 2005, 89, 4374.

(31) Garg, A. Phys. Rev. B 1995, 51, 15592.

(32) Merkel, R.; Nassoy, P.; Leung, A.; Ritchie, K.; Evans, E. Nature 1999, 397, 50 .

(33) Evans, E.; Ritchie, K. Biophys. J. 1999, 76, 2439. 
(34) Ray, C.; Brown, J. R.; Akhremitchev, B. B. J. Phys. Chem. B 2007, $111,1963$.

(35) Bell, G. I. Science 1978, 200, 618

(36) Kamholz, A. E.; Schilling, E. A.; Yager, P. Biophys. J. 2001, 80, 1967.

(37) Guo, S.; Akhremitchev, B. B. Langmuir 2008, 24, 880.

(38) Ray, C.; Brown, J. R.; Akhremitchev, B. B. Langmuir 2007, 23, 6076.

(39) Janshoff, A.; Neitzert, M.; Oberdorfer, Y.; Fuchs, H. Angew. Chem., Int. Ed. 2000, 39, 3213.

(40) Gu, C.; Ray, C.; Guo, S.; Akhremitchev, B. B. J. Phys. Chem. C 2007, 111, 12898.

(41) Kuhner, F.; Erdmann, M.; Gaub, H. E. Phys. Rev. Lett. 2006, 97, 218301

(42) Ke, C. H.; Jiang, Y.; Rivera, M.; Clark, R. L.; Marszalek, P. E. Biophys. J. 2007, 92, L76.

(43) Ratto, T. V.; Langry, K. C.; Rudd, R. E.; Balhorn, R. L.; Allen, M. J.; McElfresh, M. W. Biophys. J. 2004, 86, 2430.

(44) Ray, C.; Akhremitchev, B. B. J. Am. Chem. Soc. 2005, 127, 14739

(45) Guo, S.; Lad, N.; Ray, C.; Akhremitchev, B. B. Biophys. J. 2009, 96,3412 .
(46) Kellermayer, M. S. Z.; Bustamante, C.; Granzier, H. L. Biochim. Biophys. Acta 2003, 1604, 105.

(47) Rubinstein, M.; Colby, R. H. Polymer Physics; Oxford University Press: New York, 2003.

(48) Mattice, W. L.; Helfer, C. A.; Sokolov, A. P. Macromolecules 2003 , $36,9924$.

(49) Chen, A.; Moy, V. T. Biophys. J. 2000, 78, 2814.

(50) Oesterhelt, F.; Rief, M.; Gaub, H. E. New J. Phys. 1999, 1, 6.1.

(51) Ray, C.; Brown, J. R.; Akhremitchev, B. B. J. Phys. Chem. B 2006, $110,17578$.

(52) Ray, C.; Brown, J. R.; Kirkpatrick, A.; Akhremitchev, B. B. J. Am. Chem. Soc. 2008, 130, 10008.

(53) Dufrene, Y. F.; Hinterdorfer, P. Pflugers Arch. 2008, 456, 237.

(54) Al-Maawali, S.; Bemis, J. E.; Akhremitchev, B. B.; Leecharoen, R.; Janesko, B. G.; Walker, G. C. J. Phys. Chem. B 2001, 105, 3965.

(55) Valiaev, A.; Lim, D. W.; Schmidler, S.; Clark, R. L.; Chilkoti, A.; Zauscher, S. J. Am. Chem. Soc. 2008, 130, 10939.

(56) Kuhner, F.; Gaub, H. E. Polymer 2006, 47, 2555.

JP100543U 